\title{
You can't be a mensch and not do it ... Ein Kommentar
}

\author{
von Philip J. Davis
}

Philip J. Davis, Jahrgang 1923, ist Professor Emeritus für Angewandte Mathematik an der Brown University. Einer breiteren Leserschaft ist er durch seine in neun Sprachen übersetzten Bücher zur Philosophie der Mathematik, zur Unterhaltungsmathematik und durch seine vergnüglichen Geschichten über die philosophierende Katze Thomas Gray bekannt. Zusammen mit Reuben Hersh erhielt er 1983 den American Book Award in Science für ihr gemeinsames Werk The Mathematical

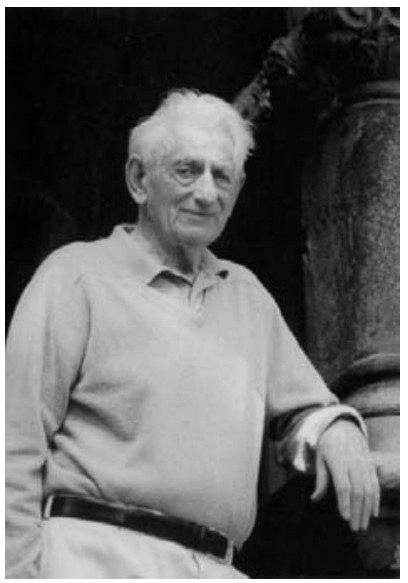
Experience (Erfahrung Mathematik, Birkhäuser 1985).

Im Verlag AK Peters sind letztes Jahr unter dem Titel The Education of a Mathematician Teile seiner Erinnerungen erschienen, in welchen er u. a. versucht, Thomas Jefferson anhand seines eigenen Werdegangs die Entwicklung der Mathematik nahezubringen.

\section{Die Claypreise ${ }^{1}$}

Vor einigen Monaten wurde die Welt forschender Mathematiker von der Ankündigung in Bann geschlagen, das Clay-Institut für Mathematik, eine private Einrichtung, habe einen Preis von sieben Millionen Dollar gestiftet: je eine Million für die Lösung von sieben berühmten mathematischen Problemen. Ausgewählt von einem Komitee verdienter Würdenträger, umfassen diese ,JJahrtausendpreisprobleme“: $P=N P$, die Riemann'sche Vermutung, die Hodge'sche Vermutung, die Poincaré'sche Vermutung, das Existenzproblem der Yang-Mills-Gleichung und die Massenlücke, Existenz und Regularität von Lösungen der Navier-Stokes-Gleichung sowie die Vermutung von Birch und Swinnerton-Dyer. Diese hochspezialisierten Probleme können einer allgemeinen Leserschaft kaum leicht erklärt werden, auch wenn sich das Clay-Institut in seiner Pressemitteilung daran versucht hat. Die Aufregung sickerte bis herunter zur breiten Öffentlichkeit und binnen weniger Stunden waren etliche wichtige E-Mail-Adressen mit abertausenden von Anfragen verstopft - weit mehr als es forschende Berufsmathematiker weltweit gibt. Als ich meinen Freund Charles informierte, der einen Doktortitel in Angewandter Mathematik trägt und viele Jahre in den Führungsstäben der Softwareindustrie gearbeitet hat, sagte er:

Toll! Wunderbar! Ein Hauch glamourösen Pfiffs ist also endlich in das Leben jener Disziplin getreten, welche dem Durchschnittsbürger so interessant erscheint wie eine Liste der Zahlen des Telefonbuchs von Manhattan. Begreift endlich, dass unser Beruf verdient, seine Bobby Fischers ${ }^{2}$ und Tiger Woodse ${ }^{3}$ zu haben.

Bobby und Tiger ungeachtet sind meine Ansichten ambivalent. Deshalb habe ich eine informelle, nicht repräsentative und nicht bindende Umfrage unter einigen mir bekannten Mathematikern durchgeführt. Ich fand diese Gruppe geteilter Meinung. Obwohl ich nicht dazu ausgebildet bin, meine Prozentzahlen auf die gesamte mathematische Gemeinschaft zu extrapolieren (könnte ich die Theorie kleiner Stichproben verwenden?), so habe ich dennoch genügend individuelle Rechtfertigungen gesammelt, pro und kontra, um diesen Artikel zu verfassen.

\section{Ein wenig Hintergrund}

Es hat schon immer Preise für mathematische Leistungen gegeben und das wird so bleiben. Der erste solche Preis, den ich kenne, geht zurück in die Zeit 585 v.Chr. Er wurde Thales von Milet verliehen, einer recht verschmommenen Gestalt, der man nachsagt, Vater der deduktiven Geometrie zu sein - ein gewaltiger Schritt in der Geschichte der Mathematik. Der Preis war für den klügsten vorhandenen Menschen geboten und Thales gewann ihn zweimal, wie Diogenes Laertius achthundert Jahre später schreibt. Welchen Preis er genau erhielt, davon überliefert Diogenes zwei Versionen. Nach der ersten gewann Thales einen Dreifuß. Der zweiten zufolge gewann er eine

\footnotetext{
1 Das Original mit dem Titel "One Million Bucks for a 100\% Solution" wurde im Oktober 2000 in den SIAM News 33 (8), pp. 6-7, gedruckt. Die Übersetzung erscheint mit freundlicher Genehmigung des Autors und der SIAM.

2 Als kleinen Service für den mit der angelsächsischen Walhalla nicht so vertrauten Leser fügen wir ein paar erklärende Fußnoten bei: Bobby Fischer $\left({ }^{*} 1943\right)$, US-amerikanischer Schachheld (Anm. des Übers.)

3 Tiger Woods (*1975), US-amerikanischer Golfheld (Anm. des Übers.)
} 
Art Pokal. Sicherlich ist keines von beiden das antike Äquivalent von einer Million Dollar.

Deutlich später wurden die drei berühmten Probleme der Antike, die Quadratur des Kreises, die Würfelverdopplung und die Dreiteilung des Winkels - drei sehr spezielle Probleme - aufgestellt; wenn auch nicht wirklich als Preiswettkampf: Das Orakel von Delos hatte prophezeit, dass sich eine Pestplage vermeiden ließe, wenn man eine Lösung dieser Probleme fände. Diese drei Probleme wurden erst vor anderthalb Jahrhunderten als ,unmöglich“ erledigt mit Hilfe mathematischer Konzepte und Resultate, welche schwerlich verfügbar waren, als das Orakel sprach. Die Pest dauert an: selbst heute werden Mathematiker mit Einsendungen von „Lösungen“ genau dieser Probleme geplagt, vorgeschlagen von Leuten mit schlichten mathematischen Fertigkeiten, welche weder die Voraussetzungen der Probleme begreifen noch die Qualitätsmerkmale einer korrekten Lösung. Ich habe meinen Anteil solcher Lösungen erhalten und vermute einmal, dass im Hinterkopf einiger dieser Einsender der Gedanke spukte, ein hoher Preis würde für die Lösung winken. Ein anderes Motiv ist die Gelegenheit, es diesen neunmalklugen Experten zu zeigen, welche sagen ,es geht nicht.“

Im Jahre 1738 schrieb die französische Académie des Sciences einen Preis für die beste Abhandlung über die Natur des Feuers aus. Der Preis wurde unter drei Leuten aufgeteilt, einer von ihnen war der große Schweizer Mathematiker Leonhard Euler (1707-1783). Wir haben mit Eulers Einsendung die Anfänge der trigonometrischen Lösung partieller Differentialgleichungen. Im Jahre 1889 gewann Henri Poincaré den König-Oscar-Preis für die Lösung des N-Körperproblems, obwohl er keine hundertprozentige Lösung besaß. (Gut gemacht, Poincaré: Regeln können stets aufgeweicht werden.) Nähern wir uns der heutigen Zeit. Der Mathematiker Paul Wohlskehl, Sohn eines Bankiers, bot in seinem Testament 1908 einen Preis von 100.000 Goldmark für die Lösung des Fermat'schen Problems. Am Rande des Selbstmords wegen einer unglücklichen Liebesaffäre hatte er psychologische Linderung darin gefunden, seine Gedanken diesem Problem zu widmen. ${ }^{4}$ Dieser Preis ist Symptom der besitzergreifenden Kraft ungelöster mathematischer Probleme.

$\mathrm{Zu}$ den hohen Preisen, welche heute für Mathematik geboten werden, gehören neben den Claypreisen der Goldbachpreis (1 Million Dollar), der Japanpreis
(50 Millionen Yen), der Wolfpreis (100.000 Dollar) und der Ostrowskipreis (150.000 Schweizer Franken). Unter ihnen sind nur die Claypreise und der Goldbachpreis für die Lösung spezifischer Probleme. Der Nobelpreis (9 Millionen Schwedische Kronen) ist natürlich der angesehenste Preis der Welt. Aber Alfred Nobel schloß die Mathematik von den preiswürdigen Gebieten aus. (Gerüchte kolportieren, dass Nobel den schwedischen Mathematiker Mittag-Leffler für eine Gefühlsentfremdung verantwortlich machte. $)^{5}$ Die Fieldsmedaille, welche nach der Auffassung vieler Mathematiker das gleiche Ansehen wie der Nobelpreis besitzt, trägt 15.000 kanadische Dollar ein. Das Ansehen eines Preises wird häufig in engerer Beziehung zur Reputation der Preisträger als der Höhe des Preisgeldes gesehen. Man denke an die olympischen Spiele im antiken Griechenland, wo Gewinner mit einem Lorbeerkranz gekrönt wurden. Oder nehmen Sie an, die Athleten profitierten von Werbeverträgen und Gagen?

Schulen, Universitäten, Gesellschaften verleihen Preise vom Kindergarten, wo jedes Kind einen Preis erhält, bis zu posthumen Preisen, die nur geehrte Tote erhalten. Unter den geringeren Preisen befinden sich, was das Preisgeld angeht, etliche von der American Mathematical Society verwaltete Preise. Jeder trägt ungefähr 4.000 Dollar ein. In Folge kürzlicher Vermächtnisse bieten die Toppreise der Society of Industrial and Applied Mathematics 20.000 Dollar und 10.000 Dollar. Bis vor einigen Jahren mußte man für unerbetene Preise keine Steuern zahlen. Dies ist nicht länger der Fall. Ich habe eine Reihe mathematischer und nationaler Preise gewonnen. Sie hinterließen keine Spur im Familienbudget.

Wenn wir schon einmal darüber nachdenken: was ist eigentlich der Unterschied zwischen einem Preis, einer Auszeichnung und einer Förderung? Alles sind Wettbewerbe. Im Wettbewerb um eine Förderung für eine Fakultät oder ein Institut kann es um Millionen gehen; eine solche Ausschreibung an Land gezogen zu haben, hat für diejenigen, welche den Antrag eingereicht haben, psychologisch häufig den gleichen Effekt wie, einen Preis zu gewinnen. Deshalb hat es schon immer Preise gegeben und das wird so bleiben. Die hier genannten Beispiele bilden nur die Spitze des Eisbergs.

The Swedes do it

The French do it

You can't be a mensch and not do it

4 Anm. des Übers.: Klaus Barner setzt sich in seinem Aufsatz „Paul Wolfskehl und der Wolfskehl-Preis“ (Mitteilungen der DMV, 1997 (3), 4-11) kritisch mit dieser Geschichte auseinander, welche wohl Alexander Ostrowski dem Autor erzählt hat.

5 Anm. des Übers.: Diese Gerüchte bezeichnen Gårding und Hörmander (Why is There No Nobel Prize in Mathematics, Math. Intell. 7 (3), 73-74, 1985) in ihrer Analyse der Frage, warum es keinen Nobelpreis für Mathematik gibt, als die „französischamerikanische Version" einer Mathematikersage. Sie gelangen zu dem nüchternen Schluß, dass der Gedanke an einen Preis für Mathematik dem guten Alfred Nobel aus ,selbstverständlichen Gründen“ gar nicht in den Sinn gekommen ist. 
Let's do it

Let's give a great big prize!

Mit der Bitte um Verzeihung an Cole Porter (1928)

\section{Argumente für die Claypreise}

Etliche der folgenden Argumente, ob nun pro oder kontra, gelten grundsätzlich für Preise; einige wenige gehören spezifisch zu den Claypreisen.

Es ist angemessen, überlegenes Talent und Exzellenz zu belohnen. Wie wir gesehen haben, stellen Preise eine Art von Belohnung dar. Preise spornen die Kreativität anderer an. Kreative Säfte werden hierdurch angeregt. Wettbewerb ist gut; Wettbewerb, sagt man, sei ein Gesetz der Natur, und verankert ihn gelegentlich im Recht.

Hohe Preise bieten exzellente Publizität für den Berufsstand des Mathematikers, sie machen ihn besser sichtbar. Sie bieten aber auch exzellente Publizität, Genugtuung, Ehre und Einfluß nicht nur für den Empfänger, sondern auch für Stifter, Ideengeber und Preiskomitee. „Wer wird geehrt? Der, welcher andere ehrt." So steht es im Buch der Propheten.

Preise zeigen der Öffentlichkeit, dass Mathematik weit davon entfernt ist, ein abgeschlossenes Kapitel zu sein, dass nicht, ,alles schon vorhanden ist und nur aus den Büchern und dem Web aufgelesen zu werden braucht." Preise machen die Öffentlichkeit auf die Existenz wichtiger ungelöster Probleme aufmerksam und damit auf die Notwendigkeit öffentlicher Unterstützung und Ermutigung.

Die Claypreise lenken die Aufmerksamkeit auf gewisse spezifische, fundamentale Probleme, ausgewählt vom Wissenschaftlichen Beirat des Clay-Insituts. Was wichtig ist, bestimmen Kriterien, welche unabhängig sind von etwa jenen der Drittmittelgeber und Forschungsgemeinschaften. Mit seiner Auswahl der Preisprobleme, Anwendungen verstoßend, macht das Clay-Institut deutlich, dass Mathematik die Königin der Wissenschaften und nicht nur die Kammerzofe ist und das dies so bleiben soll. Der Umstand, dass die Preise für sehr spezifische Probleme vergeben werden und nicht für allgemein herausragende Leistungen, verleiht Objektivität und vermeidet Subjektivität der Preisvergabe. Die Grundregeln für die Vergabe sind wohlüberlegt. Ein Beispiel:

„Um berücksichtigt zu werden, muß eine vorgeschlagene Lösung in einem referierten mathematischen
Journal weltweiten Rangs veröffentlicht und zwei Jahre lang nach der Publikation allgemein akzeptiert worden sein."

Wenn eine Lösung diese Hürden nimmt, werden weitere Überprüfungen durch eine Kommission von Experten des Fachgebiets vorgenommen. (Das sind schon recht strenge Regeln. Sie erinnern mich an die Art, in welcher der Vatikan sich auf seine Heiligen einigt. Ein Unterschied besteht darin, dass der Vatikan eine weit längere Wartezeit vor dem Vorschlag und der schlußendlichen Heiligsprechung verlangt. Auch wird im Fall der Mathematik kein Advocatus Diaboli benannt.)

\section{Argumente gegen die Preise}

Hohe Preise für notorisch schwierige Probleme ermutigen zu einem romantischen Blick auf eine mathematische Karriere. Natürlich muß die Welt stets ihre Shackletons ${ }^{6}$, Lindberghs ${ }^{7}$ und Hillarys ${ }^{8}$ haben. Aber man sollte erkennen, dass die besessene Hingabe an ein einziges Problem eine berufliche Karriere zerstören kann. Ein Beispiel basierend auf der Erfahrung eines lebensechten Mathematikers kann im Roman Onkel Petros und die Goldbach'sche Vermutung des Mathematikers und Filmemachers Apostolos Doxiadis gefunden werden.

Hohe Preise produzieren Stars, ein heute endemisches Phänomen, welches schlimmer wird. Sie betonen die Individuen und nicht die Leistungen als solche. Die Leistungen werden zu Abziehbildern: Auch wenn die mathematischen Resultate hohes Ansehen genießen, werden nicht viele Menschen, selbst Berufsmathematiker, ohne ein eingehendes Studium genau dessen, was erreicht wurde oder wie es erreicht wurde, sie lesen oder verstehen können.

Hohe Preise unterstreichen kommerzielle Werte. Theoreme werden zur Ware. Sie bringen Helden hervor, welche aus einem Teil Tiger Woods ${ }^{9}$ und einem Teil Sammy Glick ${ }^{10}$ bestehen. Ambitionierte Mathematiker werden große Probleme nicht allein deswegen ignorieren, weil damit kein Megageld verbunden ist. Auf der anderen Seite scheinen Preise das Auftauchen von Problemlösungen nicht gerade zu beschleunigen. Das Fermat'sche Problem wurde erst kürzlich gelöst, fast ein Jahrhundert nach der Stiftung des Wolfkehl'schen Preises.

Der Wettbewerbsaspekt von Preisen kann zu öffentlich bekundeter Erbitterung führen. Ich weiß um drei,

6 Sir Ernest Henry Shackleton (1874-1922), britischer Antarktisheld (Anm. des Übers.)

7 Charles Lindbergh (1902-1974), US-amerikanischer Fliegerheld (Anm. des Übers.)

8 Sir Edmund Percival Hillary (*1919), neuseeländischer Bergsteigerheld (Anm. des Übers.)

9 den hatten wir schon ... (Anm. des Übers.)

10 US-amerikanischer Romanheld und Kleingauner, welcher stets die Chance wittert, Geld zu machen. (Anm. des Übers.) 
vielleicht vier, ernste Beispiele während meiner Berufsjahre. Man erinnere sich, dass der Trojanische Krieg wegen der Entscheidung eines Wettstreits begonnen wurde. Hohe Preise können Geheimniskrämerei, Zank und Neid bestärken. Sie können das Bewußtsein für die Gemeinschaft der Mathematiker zerstören. Norbert Wiener (1894-1964), ein führender Mathematiker seiner Generation, vertrat nachdrücklich diese Ansicht. Wiener schuf den Begriff der „Kybernetik", welcher heute in der Umgangssprache in hundert verschiedenen Bedeutungen zu finden ist. Im Schreiben, in welchem er seinen Rücktritt von der National Academy of Sciences erklärte, schrieb Wiener 1941:

Je weniger von Medaillen, Preisen und dergleichen geredet wird, desto besser. Der Schmerz des erfolglosen Mitbewerbers wird nur durch den Schaden, welchen die Auszeichnung an einer schwachen oder eitlen Persönlichkeit anrichtet, aufgewogen oder die Ironie ihres Empfangs durch einen gealterten Forscher lange nachdem das Gute, das er bewerkstelligen konnte, verschwunden ist. Ich sage, sie sind, egal ob gerecht oder ungerecht verwaltet, eine Abscheulichkeit und sollten ohne Ausnahme abgeschafft werden.

Eine Million Dollar sind beträchtlich mehr, als was bislang für mathematische Preise gestiftet wurde, und „Quantität ändert die Qualität" nach einer wohlbekannten Dialektik, welche Marxisten zu zitieren lieben. Ein hoher Preise erniedrigt kleinere, er bringt die Stifter der kleineren in Verlegenheit. Für einige bedeuten die Claypreise die Vulgarisierung der Mathematik. Es gibt bessere Wege, das Geld zu nutzen, um mathematische Forschung zu fördern. Das ClayInstitut hat ein Teil seines Einkommens einer Auswahl kleinerer Preise, Stipendien, Workshops, usw. zugeteilt. Diese Aktivitäten sind sicherlich begrüßenswert, aber werden schwerlich in den Medien Aufsehen erregen.

Zwar erlauben die Regeln dem Preiskomitee, die Relevanz vorheriger nachhaltiger Resultate $\mathrm{zu}$ würdigen, so dass der Preis nicht notwendigerweise zur Gänze an die Person geht, welche den letzten Nagel einschlug. Aber wie weit muß man in der Geschichte der Mathematik zurückgehen, um nachhaltige Resultate zu identifizieren? Es wird gemunkelt, dass das erste der Probleme auf der Liste des ClayInsituts kurz vor der Lösung steht. Das $P=N P$ Problem soll gerüchteweise unentscheidbar sein, auf der Basis der ZFC-Mengentheorie. Sollen in dieser Zeit der Rechtsstreitigkeiten die Erben von Zermelo und Fraenkel in die Preissumme eingeschlossen werden? Das „C" steht für das Auswahlaxiom, welches von Zermelo und unabhängig von Erhard Schmidt formuliert wurde; sollen sich also auch die Abkömmlinge von Schmidt große Hoffnungen machen? Und die anderen tausend Arbeiter auf dem Feld, dienen auch sie dem, der nur steht und wartet? Hätte der gesamte komplexe Nexus der Mathematik von einigen wenigen Preisträgern geschaffen werden können?

Es gibt viele wichtige ungelöste Probleme in der Mathematik. Die internationale Mathematikerunion hat kürzlich ein Buch gesponsert (Mathematics: Frontiers and Perspectives), welches wenigstens hundert von ihnen nennt. Die Festlegung des Clay-Instituts schafft eine imaginäre Dichotomie zwischen den ,wirklich wichtigen Problemen" und anderen Problemen. Ein subjektives Urteil entscheidet darüber, welche Probleme langfristig fruchtbarer sein dürften. Niemand hätte zu Beginn des 20. Jahrhunderts voraussagen können, dass die wichtigste mathematische Entwicklung des Jahrhunderts der digitale Computer sein würde, insofern er das Leben aller betroffen hat.

Dann ist da die Frage, was einen mathematischen Beweis ausmacht. Was ist eine Lösung, gar eine vollständige Lösung? Formalisten, Logiker denken, sie wüssten es. Aber sie wissen es nicht, nicht wirklich. Man nehme als Beispiele das berühmte Vierfarbenproblem. Als der computerunterstützte Beweis bekannt wurde, wurde das Resultat von einer gewissen Gruppe von Mathematikern zurückgewiesen. Oder man betrachte Beweise, welche extrem lang sind von bis zu 5000 Seiten habe ich gehört. Welches Komitee kann sich durch soviele Seiten arbeiten ohne einzuschlafen? Es ist schlimmer als die Unmenge von Beweismaterial, welche ein Sonderermittler in Washington vorgelegt hat. Eine Lösung ist deshalb das, was die mathematisch gebildete Öffentlichkeit zu einer Lösung erklärt. Ich bringe diese Ansicht die ganze Zeit über unter die Leute, aber vermutlich wird es den Wissenschaftlichen Beirat schockieren, wenn sie begreifen, dass ihre Grundregeln antiplatonisch sind. Die Geschichte der Mathematik birgt Überraschungen für uns.

Mein eigener Haupteinwand gegen die Claypreise ist, dass sie die Natur der mathematischen Forschung verzerren, von bestehenden Startpunkten in Freiheit des Denkens zu Resultaten zu gelangen, welche in irgendeiner Weise fruchtbar erscheinen. Ursprüngliche Ziele brauchen für das spätere Werk nicht länger relevant zu sein. Die Claypreise verzerren das öffentliche Verständnis von Mathematik, indem sie das Lösen von Problemen auf Kosten des Bildens, der Ausarbeitung und Anwendung von Konzepten betonen. Jawohl, Mathematiker beginnen mit der Spezifizierung spezifischer Probleme, aber am Ende des Tages gibt es keine anderen langfristigen Ziele für die Mathematik, als fruchtbar zu sein, zu wachsen und sich die Unterstützung der Gemeinschaft zu verdienen. In diesem Zusammenhang zitiere ich die Antwort, welche ich von einem Wissenschaftshistoriker erhielt: 
Es ist eine riesige Erleichterung, dass mein Gebiet, die Geschichte, keine solchen Preise besitzt. Meiner Ansicht nach, gerade nach der Lektüre von Elisabeth Crawfords Arbeit über die Geschichte der Nobelpreise [welche nicht für vorgegebene Probleme vergeben werden], ist ihr Effekt, etwaige Forschung und die Wertschätzung derselben zu verzerren und zu verengen.

\section{Ein abschließender Gedanke}

Wenn wie in der griechischen Antike eine Stiftung einen Preis für den „Klügsten von allen“ ausschriebe, würden wir dann alle als Resultat des Wettstreits klü- ger werden? Würde die Evolution damit einen Riesenschritt vorankommen?

Adresse des Autors

Prof. Dr. Philip J. Davis

Division of Applied Mathematics

Brown University

Providence, RI 02912, USA

Philip_Davis@brown.edu

\section{Der schwierigste Code aller Zeiten ist geknackt}

\section{von Folkmar Bornemann}

Nach seinem Buch über Fermats letzten Satz gelang Simon Singh vor zwei Jahren mit The Codebook (dt. unter dem Titel Geheime Botschaften, Hanser, 2000) gleich der nächste Besteller. Er fügte diesem Buch eine Art Übungsaufgabe bei: zehn Geheimtexte aufsteigenden Schwierigkeitsgrades, genannt The Cipher Challenge, oder noch werbewirksamer, „der schwierigste Code aller Zeiten“. Singh stiftete 10.000 Pfund für die erste vollständige korrekte Lösung bis zum 1. Januar 2010.

Fünf schwedische Informatiker ${ }^{1}$ schafften es dann schon am 5. Oktober 2000. Für die Entschlüsselung des zehnten, schwierigsten Geheimtextes faktorisierten sie in 13 Tagen Laufzeit auf einer Workstation mit 4 Alpha-Prozessoren folgenden 512bit RSAModul (155 Dezimalstellen):

1074278829126656590717841127994211661266392179475 3294588877817210355464150980121879033832926235281 0907506720835049419964331434255583344018558089894 $26892463=128442051653810314916622590289775531$ 98964984323915864368216177647043137765477 • 83639 1832187606937820650856449710761904520026199724985 596729108812301394489219

Allein diese Leistung ist schon ein enormer Erfolg. Hierzu hatten sie die Implementierung des General Number Field Sieve weiterentwickelt, welche Monate zuvor am niederländischen CWI zur Rekordfaktorisierung von RSA-155 verwendet worden war. Der 38seitige, exzellente und spannende Bericht der Fünf findet sich im Internet unter http://codebook.org und beschreibt auch die Lösung der vorangehenden neun Geheimtexte (unter den Verschlüsselungsmethoden finden sich neben RSA und DES auch historisch interessante wie Playfair und eine 3-RotorEnigma).

Singh berichtet auf der Webseite der Cipher Challenge, dass sich Menschen aller Lebensbereiche und aller Alterstufen beteiligt hätten, von Anfängern und Schulkindern bis hin zu Mathematikern und Berufskryptographen, ja sogar ein Träger der Fields-Medaille sei dabei gewesen (http://www . 4thestate.co.uk/CipherChallenge).

Lehrreich ist auch ein Blick auf die Berichterstattung in der Presse, etwa im Tagesspiegel (http: //www2.tagesspiegel.de/archiv/2001/01/13/ ak-in-13184.html): die Interpretationen müssen mit Vorsicht genossen werden, da etwa Schlüssellängen symmetrischer und asymmetrischer Verfahren umstandslos in einen Topf geworfen werden.

1 Gunnar Andersson, Lars Ivansson und Staffan Ulfberg promovierten letztes Jahr in Theoretischer Informatik am Royal Institute of Technology in Stockholm, Torbjörn Granlund besitzt eine Firma für Open-Source-Softwarelösungen, Fredrik Almgren arbeitet in einer Firma für mobile Internetlösungen. 